\title{
Elexacaftor-tezacaftor-ivacaftor- A Combination Therapy for Phe508del Cystic Fibrosis
}

\author{
Shijing Jia ${ }^{1}$ and Jennifer L Taylor-Cousar ${ }^{2}$ \\ 1. University of Michigan, Ann Arbor, MI, USA; 2. National Jewish Health, Denver, CO, USA
}

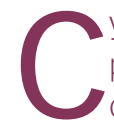
ystic fibrosis is an autosomal recessive genetic disease that causes systemic disease manifestations including, most commonly, progressive airway destruction and pancreatic insufficiency. Therapies have traditionally centered on treatment of the ramifications of organ damage as they occur. However, within the last decade, cystic fibrosis transmembrane conductance regulator (CFTR) modulator therapies have targeted the cellular pathophysiology of the disease. This review aims to highlight the advancement of this class of therapeutics, with particular focus on the newest drug approved in 2019, the triple combination modulator drug: elexacaftor-tezacaftor-ivacaftor

\section{Keywords}

Cystic fibrosis, CFTR modulators, elexacaftor, $\mathrm{VX}-445$, triple combination

Disclosure: Jennifer L Taylor-Cousar reports grants and personal fees from Vertex, personal fees from Gilead, grants and personal fees from Proteostasis, grants from Eloxx, personal fees from Protalix, grants and personal fees from Celtaxys, and personal fees from Santhera. Shijing Jia has nothing to declare in relation to this article.

Review Process: Double-blind peer review.

Compliance with Ethics: This study involves a review of the literature and did not involve any studies with human or animal subjects performed by any of the authors.

Authorship: The named authors meet the International Committee of Medical Journal Editors (ICMJE) criteria for authorship of this manuscript, take responsibility for the integrity of the work as a whole, and have given final approval for the version to be published.

Received: December 10, 2019

Accepted: January 20, 2020

Citation: US Respiratory \& Pulmonary

Diseases. 2019:4(1):15-9

Corresponding Author: Shijing Jia, University of Michigan, 1500 E. Medical Center Dr. SPC 5360, Ann Arbor, MI 48109-5360 USA. E: sjia@med.umich.edu

Support: No funding was received in

the publication of this article.
Cystic fibrosis (CF), an inherited disease affecting all races and ethnicities, results from defects in the cystic fibrosis transmembrane conductance regulator (CFTR) gene, which leads to dysregulations in the ion and fluid balance across epithelial membranes throughout the body. ${ }^{1-3}$ The CFTR protein is an anion channel that functions as a transmembrane traffic ATPase which is regulated by CAMP and protein kinase A phosphorylation. ${ }^{4,5}$ Due to the prevalent nature of the CFTR channel in epithelial cells, CF-causing mutations lead to multisystemic disease processes. At present, an estimated 80,000 people live with CF worldwide, with over 30,000 within the United States. ${ }^{6,7}$ Because CF is inherited in an autosomal recessive way, significant portions of the population ( 10 million in the USA) are asymptomatic carriers of disease-causing mutations. ${ }^{6,8}$ The most common disease-causing mutation is Phe508del (F508del), a deletion of the phenylalanine at the 508 position of the protein. ${ }^{6}$ However, over 2,000 mutations or variants have been identified in the CFTR gene, some known to be disease causing, while the impacts of most are uncertain, and some occurring only in a handful of people with $\mathrm{CF}^{9}$

In order to conceptualize the vast numbers of CFTR genetic variants and the clinical ramifications, disease-causing mutations have been classified into six categories. ${ }^{10-12}$ Class I mutations cause severely decreased levels of total protein production due to premature termination of transcription, such as in the case of nonsense mutations and certain splice site mutations or deletions. Class II mutations account for a large majority of CF-causing variants and include the common F508del variant. Class II variants are considered processing mutations that cause misfolding of the CFTR protein; cellular clearance of the abnormal protein in the endoplastic reticulum results in a deficient number of mature CFTR protein successfully trafficked to the cellular surface. Class III mutations are known as gating mutations in which the primary defect is the inability of the CFTR channel pore to open appropriately and thus results in lack of ion transfer in spite of appropriate channel location at the cell surface. Class IV mutations are similar to class III mutations and are conduction mutations which have reduced overall conductance across the channel. Class V mutations have decreased total protein production due to mutations with altered promoter regions or splice sites. Class VI mutations have instability of the formed CFTR protein at the membrane and as a result cause increased turnover of functional protein and thus decreased total ion transport.

Since the description of this classification system, it has been recognized that many mutations exhibit more than one type or class of defect.12 Thus a drug that is able to correct any portion of the defects commonly observed in the CFTR protein may be beneficial for more than a single mutation class. More physiologic and clinically meaningful classifications have been proposed and are often used to describe classes of CFTR mutations. For example, residual function mutations are those CFTR variants which enable some CFTR protein to be expressed at the cell surface versus minimal function (MF) mutations where there is little to no CFTR modulator-responsive protein at the cell surface. As a 
Table 1: Summary of phase III clinical trials of CFTR modulators in people with cystic fibrosis age $\geq 12$ years

\begin{tabular}{|c|c|c|c|c|c|c|}
\hline Modulator & Mutation & $\Delta \mathrm{ppFEV}_{1}$ & PEX RRR & $\Delta$ weight & $\Delta \mathrm{CFQ}-\mathrm{R}$ & $\Delta \mathrm{SWCl}(\mathrm{mmol} / \mathrm{L})$ \\
\hline Iva vs placebo ${ }^{21}$ & G551D & $+10.6 \%$ & $55 \%$ & $+2.8 \mathrm{~kg}$ & +8.6 & -48.1 \\
\hline Lum/iva vs placebo ${ }^{36}$ & F508del/F508del & $+2.8 \%$ & $39 \%$ & $+0.24 \mathrm{~kg} / \mathrm{m}^{2}$ & $+2.2^{*}$ & NDA \\
\hline $\begin{array}{l}\text { Tez/iva vs placebo } \\
\text { Iva vs placebo }{ }^{26}\end{array}$ & F508del/RF & $\begin{array}{l}+6.8 \% \\
+4.7 \%\end{array}$ & $\begin{array}{l}46 \% * \\
54 \% *\end{array}$ & $\begin{array}{l}+0.16 \mathrm{~kg} / \mathrm{m}^{2 *} \\
+0.29 \mathrm{~kg} / \mathrm{m}^{2 *}\end{array}$ & $\begin{array}{l}+11.1 \\
+9.7\end{array}$ & $\begin{array}{l}-9.5 \\
-4.5\end{array}$ \\
\hline Elexa/tez/iva vs tez/iva ${ }^{51}$ & F508del/F508del & $+10.0 \%$ & NDA & NDA & +17.4 & -45.1 \\
\hline
\end{tabular}

Data displayed is as compared to control group at the conclusion of each study.

*Not statistically significant.

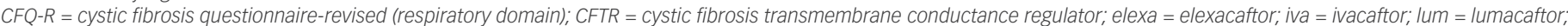
$M F=$ minimal function; NDA = no data available; $P E X=$ pulmonary exacerbation; $p p F E V_{1}=$ percent predicted forced expiratory volume in 1 second; $R F=$ residual function; $R R R=$ relative risk reduction; $\mathrm{SWCl}=$ sweat chloride; te $=$ tezacaftor.

result, a drug that improves function of CFTR channel would be effective in the case of residual function mutations, but would not be effective for the majority of MF mutations.

The hope for a drug therapy that targets CF pathology at the cellular level is a drastic improvement over traditional CF care. Prior to the advent of CFTR modulators, therapeutic interventions were aimed at decreasing and managing the manifestations of the disease. Inhalation of hypertonic saline and dornase alfa thinned sputum to improve airway clearance; antibiotics were used to treat pulmonary infections; pancreatic enzymes were prescribed to replace missing digestive enzymes; and insulin was increasingly required as people with CF eventually developed pancreatic endocrine insufficiency after years of pancreatic duct mucoid plugging, causing islet cell destruction. ${ }^{13}$ Instead of palliating measures after the development of disease manifestations, modulators target the root cause of CF by correcting the CFTR protein dysfunction at the cellular level.

\section{Single- and dual-combination CFTR modulators}

The first clinically available CFTR modulator was ivacaftor (VX-770), initially approved by the US Food and Drug Administration (FDA) in 2012. ${ }^{14}$ Ivacaftor is a small-molecule potentiator that binds directly to the CFTR protein and increases the time in which the channel is in the open configuration. ${ }^{15-17}$ While the clinical benefit is most pronounced in people with CF with primary gating mutations, the drug improves chloride transport in a wide variety of CFTR mutations including in the normal protein state. ${ }^{15,18,19}$ In pre-clinical studies, ivacaftor increased CFTRdependent ion transport in cultured human bronchial epithelial cells with either G551D or F508del mutations. ${ }^{19}$ Subsequent clinical studies yielded remarkable results. ${ }^{20,21}$ In a randomized phase III trial in people with CF with a G551D gating mutation, compared to placebo, ivacaftor dramatically improved the percent predicted forced expiratory volume in 1 second $\left(\mathrm{ppFEV}_{1} ;+10.6 \%, \mathrm{p}<0.001\right)$, respiratory symptom scores as measured by the Cystic Fibrosis Questionnaire-revised (CFQ-R, +8.6 points, $p<0.001$, minimal clinically important difference $=4.0),{ }^{22}$ weight $(+2.7$ kilograms, $p<0.001)$, and decreased rates of exacerbations $(-55 \%$, $\mathrm{p}<0.001)^{21}$ (Table 1 and Figure 1). Since initial FDA approval, ivacaftor's indication has been expanded to other gating or conductance mutations with residual function of CFTR based on clinical and non-clinical studies. ${ }^{15,23-26}$ This transformative approach to rapid drug approval speaks to the importance of the strong foundation of understanding of $\mathrm{CF}$ pathophysiology, the pre-clinical data in support of clinical benefit, and
Figure 1: Results of phase III clinical trials of CFTR modulators

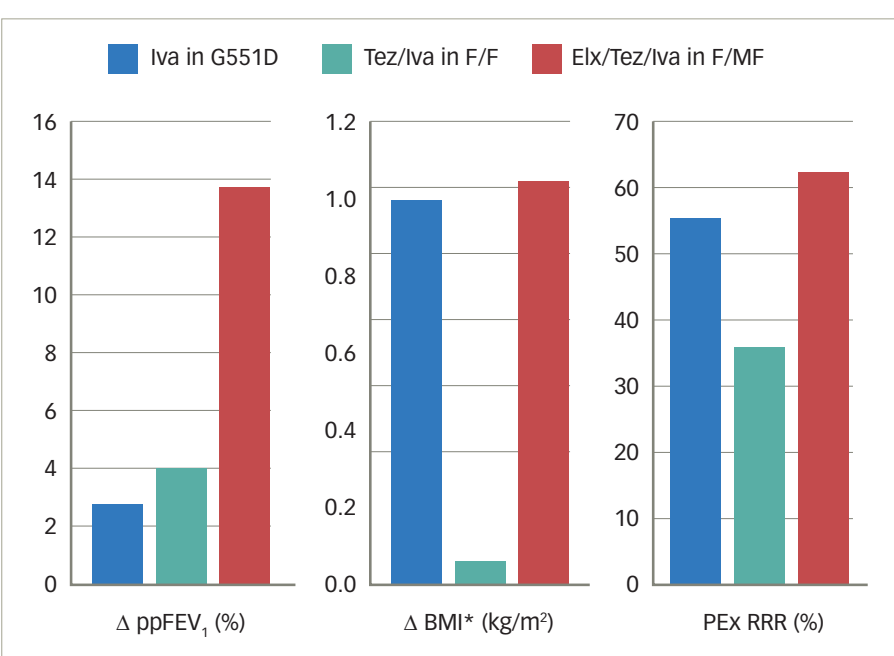

${ }^{*}$ Change in BMI of ivacaftor in G551D was estimated using published data of change in total weight in kilograms.

$B M I=$ body mass index; $C F T R=$ cystic fibrosis transmembrane conductance regulator; el $x$ = elexacaftor; iva = ivacaftor; lum = lumacaftor; $M F$ = minimal function; $P E X=$ pulmonary exacerbation; pPFEV $_{1}=$ percent predicted forced expiratory volume in 1 second; $R R R=$ relative risk reduction; tez = tezacaftor

the sense of urgency to bring highly effective treatments to people with a life-shortening disease.

The benefits seen in the initial clinical studies of ivacaftor are sustained over time. People with CF who have been prescribed ivacaftor have significantly lower mortality, need for lung transplantation, hospitalizations, and pulmonary exacerbations over time. ${ }^{27}$ While ivacaftor proved clearly to be a highly effective therapy, people with CF who could benefit with gating and residual function mutations represented a small fraction (13.6\%) of those with CF. ${ }^{6}$ Unfortunately, the use of a potentiator alone did not improve CFTR function sufficiently in people homozygous for the F508del mutation to provide clinical benefit. ${ }^{28}$ As a class II mutation, CFTRF508del results in several protein processing defects, including protein folding, trafficking, and cell surface turnover. ${ }^{29}$ While CFTR-F508del seems to also have conformational defects leading to gating abnormalities, the singular effect of gating correction is not sufficient to restore function in a clinically meaningful quantity due to a lack of total ion channels 
successfully transported to the cellular membrane. Although the frequency of the F508del mutation varies across populations, because it is the most common mutation with $84.7 \%$ of people with CF in the USA carrying at least one copy, the focus of CFTR modulator development turned to correctors, drugs that could increase the number of ion channels on the epithelial cell surface. .30

Lumacaftor (VX-809) was the first CFTR corrector to be approved for clinical use in the USA in combination with ivacaftor in 2015..$^{31}$ Lumacaftor works by improving CFTR-F508del processing in the endoplasmic reticulum leading ultimately to higher fractions of the defective protein arriving at the epithelial cell surface. ${ }^{32,33}$ Because CFTR-F508del proteins also exhibit gating defects, lumacaftor in isolation does not rescue the protein in a clinically meaningful way. ${ }^{3,35}$ However, when used in combination with the gating potentiator ivacaftor, dual drug therapy improved ppFEV ${ }_{1}(+2.6 \%$ to $+4.0 \%, p<0.001)$ and decreased pulmonary exacerbations $(-30 \%$ to $-39 \%, p<0.001$ ) in people with CF homozygous for F508del. 36,37 While these results were not as dramatic as in the case of ivacaftor in those with CF with gating mutations, a 2-year extension study of the trial showed a $42 \%$ slower rate of ppFEV $_{1}$ decline over time. ${ }^{38}$ As predicted by the relatively modest level of CFTR correction in vitro, the dual combination CFTR modulator did not show efficacy in people with CF heterozygous for F508del and a MF mutation. ${ }^{39}$

Tezacaftor (VX-661) is chemically similar to lumacaftor and became the next CFTR modulator available, in combination with ivacaftor, to people with CF in the USA (2018). ${ }^{40}$ As a CFTR corrector, tezacaftor also improves protein folding and trafficking to the epithelial cell surface, but provides the benefit over lumacaftor in not being an inducer of CYP3A4 enzymes and thus exhibits fewer drug-drug interations. ${ }^{41}$ Additionally, tezacaftor was not associated with increased chest tightness, a problem seen with lumacaftor. Clinical studies of dual combination tezacaftor-ivacaftor show favorable results, with $a+4.0 \%$ improvement in $\mathrm{ppFEV}_{1}(\mathrm{p}<0.001)$ and $-35 \%$ lower rate of pulmonary exacerbations $(\mathrm{p}=0.005){ }^{42}$ In people who were heterozygous for F508del and a residual function mutation, including those who were then not eligible for ivacaftor, a parallel group study evaluated the efficacy of tezacaftor-ivacaftor combination and ivacaftor alone in additional mutations shown to be responsive in vitro. ${ }^{39}$ Tezacaftor-ivacaftor improved $\operatorname{ppFEV}_{1}(+6.8 \%, p<0.001)$.

While the advent of combination corrector-potentiator modulator therapy served as a proof of concept for the potential to rescue the CFTR-F508del protein, tezacaftor and lumacaftor were not considered highly effective therapies, with only modest clinical benefits when compared to ivacaftor alone in people with responsive gating mutations. Additionally, a significant portion $(\sim 42 \%)$ of people with CF remained ineligible for any modulator therapy. ${ }^{628,39}$ For a highly efficacious treatment that would impact more people with $\mathrm{CF}$, there was more work to be done.

\section{A triple combination CFTR modulator}

In spite of the use of tezacaftor, a more or synergistically effective modulator for trafficking CFTR-F508del protein to the cell surface was needed. The primary target for therapeutic drug development was an effective corrector that would be complementary to the available dual combination therapies. With high throughput drug discovery, several agents were identified as potential targets for development.. ${ }^{43}$
Four next-generation CFTR correctors (VX-152, VX-440, VX-445 or elexacaftor, and VX-659) were evaluated in parallel phase I and II studies in people with CF with either two copies of F508del or one copy of F508del and a MF mutation. .3.4.4 $^{43}$ While initial clinical results were remarkably promising for all four compounds, based on efficacy and side effect profiles, VX-445 and VX-659 were selected to be brought to the next stage of clinical testing. ${ }^{4345-47}$ The detailed trial design and results of the VX-445 (elexacaftor) and VX-659 preclinical and early clinical studies were published. ${ }^{46,47}$ In vitro CFTR protein processing and trafficking in airway epithelial cells isolated from people with CF with either F508del homozygous or F508del/MF mutations as assessed by quantity and function of mature CFTR protein, were significantly higher in the presence of VX-445 and VX-659 in combination with tezacaftor and ivacaftor. Both drugs were assessed in two groups of subjects. Critical for consideration of study design, people with CF who were homozygous for F508del mutations had both tezacaftor-ivacaftor and lumacaftor-ivacaftor clinically available for use. Because of the favorable pharmacology (drug-drug interactions), tezacaftorivacaftor was chosen as the backbone of triple combination (TC) therapy. In people with CF with F508del and a MF mutation, who had no modulatory therapy clinically available, the investigational drugs in TC with tezacaftorivacaftor were compared to placebo. However, in subjects homozygous for F508del mutations, the drug in TC with tezacaftor-ivacaftor was compared to dual combination tezacaftor-ivacaftor as the control.

Clinical studies demonstrated favorable safety and efficacy profiles consistent with the in vitro CFTR activity improvement. In phase II trials, triple therapy with $\mathrm{VX}$-445-tezacaftor-ivacaftor was associated with an increase in $\mathrm{ppFEV}_{1}$ of $13.8 \%(p<0.001)$ in the F508del/MF subjects. ${ }^{46}$ In subjects homozygous for F508del mutations, addition of the third drug VX445 to tezacaftor-ivacaftor led to an increase in ppFEV ${ }_{1}$ of $11.0 \%(p<0.001)$ on top of that attributable to tezacaftor-ivacaftor alone. Similarly, VX-659tezacaftor-ivacaftor triple therapy increased ppFEV ${ }_{1}$ by $13.3 \%(p<0.001)$ in the F508del/MF subjects compared to placebo, and by $9.7 \%(p<0.001)$ in the F508del homozygous subjects compared to dual tezacaftor-ivacaftor therapy. ${ }^{47}$ This degree of clinical response to the TC drugs surpassed those of ivacaftor in early clinical trials in people with CF with the G551D mutation, sparking much enthusiasm in the CF community.

In rapidly enrolled phase III studies, VX-659 and VX-445 were evaluated in TC against placebo in subjects with the F508del/MF mutations and against tezacaftor-ivacaftor in subjects homozygous for F508del: AURORA (NCT03525444, NCT03525548 and NCT03525574) and ECLIPSE (NCT03447249, NCT03460990 and NCT03447262). ${ }^{43}$ In both protocols, the TC was evaluated over 24 weeks in subjects with F508del/MF mutations with the recruitment goal powered to evaluate for significant differences in both changes in ppFEV 1 at 4 and 24 weeks, as well as pulmonary exacerbation rates through 24 weeks. However, because USA subjects homozygous for F508del had available dual combination CFTR modulators, the study for this group of subjects was designed for a duration of 4 weeks, powered for change in ppFEV ${ }_{1}$ only. Because blinded safety data could be obtained from the 48-week studies in subjects heterozygous for F508del/MF mutations, and the change of ppFEV 1 at 4 weeks in previous modulator studies has been sustained at 24- and 48-week extension studies, the 4-week endpoint

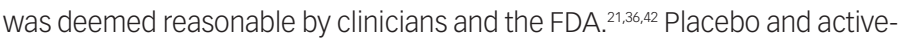
controlled arms for both VX-445 and VX-659 were rolled into 96-week openlabel extension studies to allow study participants access to drug as well as to gather additional safety data (NCT03525574 and NCT03447262). 
Figure 2: Estimated proportions of people with cystic fibrosis in the USA eligible for CFTR modulator therapy in 2012, 2015, and 2019

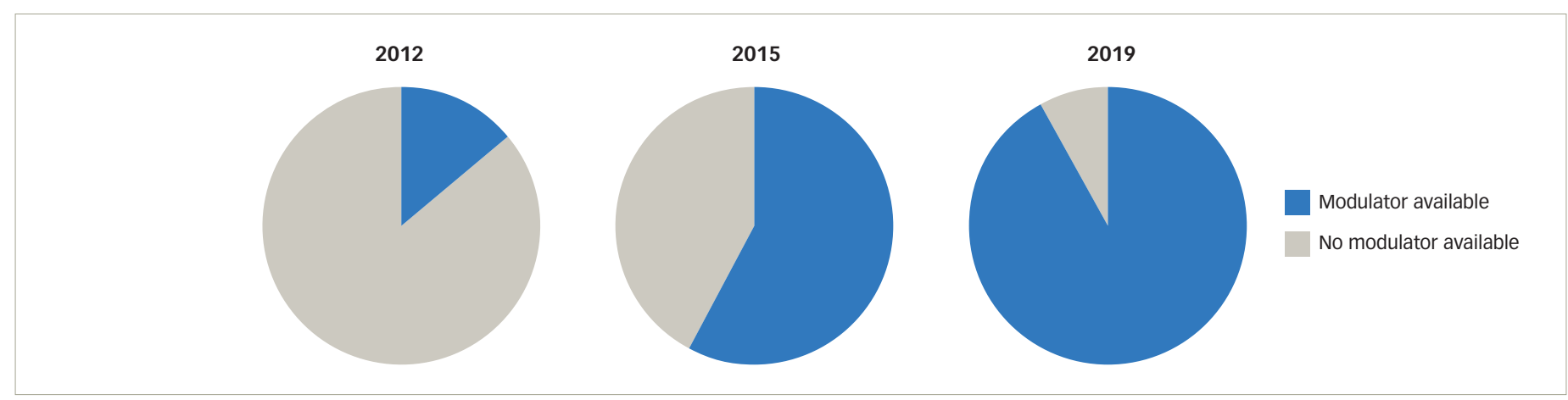

CFTR = cystic fibrosis transmembrane conductance regulator.

The phase III study for VX-659-tezacaftor-ivacaftor was completed first, with interim results released in November 2018 showing a dramatic ppFEV ${ }_{1}$ improvement of $14.0 \%$ ( $\left.p<0.0001\right)$ compared to placebo in subjects with F508del/MF mutations, and a 10.0\% ( $p<0.0001)$ improvement when VX-659 was added to tezacaftor-ivacaftor in subjects homozygous for F508del mutations at 4 weeks. ${ }^{48}$ Full study results including incremental improvements in pulmonary exacerbation rates, pulmonary symptom scores, and nutritional status were not available at that time.

VX-445 (elexacaftor)-tezacaftor-ivacaftor performed similarly based on interim results announced in March 2019. ${ }^{49}$ Subsequently, complete study results were published in both subsets of subjects - those homozygous for F508del and those heterozygous for F508del and a MF mutation. In subjects heterozygous for F508del and a MF mutation without prior modulator therapy available, elexacaftor-tezacaftor-ivacaftor improved ppFEV 1 13.8\% $(p<0.001)$ at week 4 and $14.3 \%(p<0.001)$ at week 24 compared to placebo in 403 subjects. ${ }^{50}$ The rate of annualized pulmonary exacerbations at 24 weeks was $63 \%$ lower compared to placebo $(p<0.001)$. Sweat chloride concentrations improved $-41.8 \mathrm{mmol} / \mathrm{L}$ compared to placebo at 24 weeks $(p<0.001)$. CFQ-R RD scores at 24 weeks improved 20.2 points compared to placebo ( $p<0.001)$. BMI improved $1.04 \mathrm{~kg} / \mathrm{m}^{2}$ at 24 weeks ( $\left.p<0.001\right)$, with an absolute increase of $2.9 \mathrm{~kg}$ (95\% confidence interval 2.3-3.4), as compared to placebo. These results demonstrated that elexacaftor-tezacaftorivacaftor was highly effective in subjects with only one F508del mutation.

The TC drug was also evaluated in subjects homozygous for F508del over 4 weeks as compared to dual combination tezacaftor-ivacaftor therapy. ${ }^{51}$ On top of the impact of tezacaftor-ivacaftor, addition of VX-445 (elexacaftor) improved mean $\mathrm{ppFEV}_{1}$ by $10.0 \%$ ( $\left.\mathrm{p}<0.0001\right)$, sweat chloride concentration by $-45.1 \mathrm{mmol} / \mathrm{L}(\mathrm{p}<0.0001)$, and CFQ-R RD scores by 17.4 points $(p<0.0001)$. Combined safety events from both patient groups demonstrated that most side effects reported during the study were mild or moderate. ${ }^{50,51}$ Almost all adverse events were equally reported in the control or placebo groups compared to those receiving the TC drug. The events with a higher signal in the elexacaftor-tezacaftor-ivacaftor arm included diarrhea (13\% in TC group versus $7 \%$ in placebo), abdominal pain (14\% versus 9\%), rash (10\% versus 5\%), and increases in liver enzymes (alanine aminotransferase (ALT), aspartate transaminase (AST), bilirubin: $5-10 \%$ versus $1-3 \%) .{ }^{52}$ Based on the results of these phase III studies, the FDA approved TC elexacaftortezacaftor-ivacaftor through fast-track and orphan drug study status for use in people with $C F \geq 12$ years of age with at least one copy of F508del on October 21, 2019 in a record 62 days after application. ${ }^{52-54}$

\section{Future directions}

For the first time since CF was described by Dr Dorothy Andersen in 1938, 55 a highly effective therapy (either ivacaftor alone for those heterozygous for ivacaftor-responsive mutations or elexacaftor-tezacaftor-ivacaftor for those with at least one F508del mutation), which targets the underlying cause of the progressive multi-systemic disease, has the potential to change the clinical course for approximately 90\% of people with CF (Figure 2). Unfortunately, a disproportionate number of racial and ethnic minorities do not have an F508 allele and will thus be ineligible for this new therapy.56 Nonetheless, elexacaftor-tezacaftor-ivacaftor is currently being evaluated for use in children with CF aged 6-11 (NCT03691779), and it is expected that demonstration of efficacy and safety in this age group will be followed by testing in younger age groups. It is hypothesized that initiation of highly effective CFTR modulator therapy from the time of diagnosis of CF by newborn screening may prevent the complications of the disease and dramatically change the face of CF.

The $\sim 10 \%$ of the USA CF population not expected to respond to CFTR modulator therapy predominantly consists of those people with CF with mutations leading to insufficient quantities of mature CFTR protein resulting from premature stop mutations, splice site mutations, and mutations that destabilize the protein at the membrane surface. ${ }^{6,12}$ Approaches to therapy for this group of people with CF include CFTR amplifiers, read-through therapies, RNA modifying agents, and approaches to direct DNA repair. Clinicians, investigators, and the entire CF community continue to hope that this substantial step forward in the treatment of this life shortening disease will be followed by a cure for all people with CF. $\square$ 
1. Riordan JR, Rommens JM, Kerem B, et al. Identification of the cystic fibrosis gene: cloning and characterization of complementary DNA. Science. 1989;245:1066-73.

2. Rommens JM, lannuzzi MC, Kerem B, et al. Identification of the cystic fibrosis gene: chromosome walking and jumping. Science. 1989;245:1059-65.

3. Kerem B, Rommens JM, Buchanan JA, et al. Identification of the cystic fibrosis gene: genetic analysis. Science. 1989;245: 1073-80

4. Anderson MP, Gregory RJ, Thompson S, et al. Demonstration that CFTR is a chloride channel by alteration of its anion selectivity. Science. 1991;253:202-5.

5. Liu F, Zhang Z, Csanady L, et al. Molecular structure of the human CFTR ion channel. Cell. 2017:169:85-95.e88.

6. Cystic Fibrosis Foundation. 2018 Patient Registry Annual Data Report. 2019. Available at: www.cff.org/Research/ResearcherResources/Patient-Registry/2018-Patient-Registry-Annual-DataReport.pdf (accessed January 23, 2020).

7. Zolin A, Orenti A, Naehrlich L, et al. ECFS Patient Registry Annual Data Report 2017. 2019. Available at: www.ecfs.eu/sites/default/ files/general-content-images/working-groups/ecfs-patientregistry/ECFSPR_Report2017_v1.3.pdf (accessed January 23, 2020).

8. Strom CM, Crossley B, Buller-Buerkle A, et al. Cystic fibrosis testing 8 years on: lessons learned from carrier screening and sequencing analysis. Genet Med. 2011;13:166-72.

9. Clinical and Functional Translation of CFTR, 2019. Available at www.cftr2.org/ (accessed January 23, 2020).

10. Rowe SM, Miller S, Sorscher EJ. Cystic fibrosis. N Eng/ J Med. 2005:352:1992-2001.

11. Sosnay PR, Siklosi KR, Van Goor F, et al. Defining the disease liability of variants in the cystic fibrosis transmembrane conductance regulator gene. Nat Genet. 2013;45:1160-7.

12. Veit G, Avramescu RG, Chiang AN, et al. From CFTR biology toward combinatorial pharmacotherapy: expanded classification of cystic fibrosis mutations. Mol Biol Cell. 2016;27:424-33.

13. Castellani C, Duff AJA, Bell SC, et al. ECFS best practice guidelines: the 2018 revision. J Cyst Fibros. 2018:17:153-78.

14. Vertex Pharmaceuticals Inc. Kalydeco (ivacaftor) [package insert] Cambridge, MA. 2012

15. Yu H, Burton B, Huang CJ, et al. Ivacaftor potentiation of multiple CFTR channels with gating mutations. J Cyst Fibros. 2012;11:237-45

16. Liu F, Zhang Z, Levit A, et al. Structural identification of a hotspot on CFTR for potentiation. Science. 2019;364:1184-8.

17. Yeh HI, Qiu L, Sohma Y, et al. Identifying the molecular target sites for CFTR potentiators GLPG1837 and VX-770. J Gen Physiol. 2019;151:912-28.

18. Cui G, Stauffer BB, Imhoff BR, et al. VX-770-mediated potentiation of numerous human CFTR disease mutants is influenced by phosphorylation level. Sci Rep. 2019;9:13460.

19. Van Goor F, Hadida S, Grootenhuis PD, et al. Rescue of CF airway epithelial cell function in vitro by a CFTR potentiator, VX-770. Proc Natl Acad Sci USA. 2009;106:18825-30.

20. Accurso FJ, Rowe SM, Clancy JP, et al. Effect of VX-770 in person with cystic fibrosis and the G551D-CFTR mutation. N Eng/ I Med. 2010:363:1991-2003.

21. Ramsey BW, Davies J, McElvaney NG, et al. A CFTR potentiator in patients with cystic fibrosis and the G551D mutation. N Eng/ Med. 2011;365:1663-72.

22. Quittner AL, Buu A, Messer MA, et al. Development and validation of The Cystic Fibrosis Questionnaire in the United States: a health-related quality-of-life measure for cystic fibrosis. Chest. 2005; 128:2347-54.
23. Moss RB, Flume PA, Elborn IS, et al. Efficacy and safety of ivacaftor in patients with cystic fibrosis who have an Arg117HisCFTR mutation: a double-blind, randomised controlled trial. Lancet Respir Med. 2015;3:524-33.

24. De Boeck K, Munck A, Walker S, et al. Efficacy and safety of ivacaftor in patients with cystic fibrosis and a non-G551D gating mutation. J Cyst Fibros. 2014:13:674-80.

25. Davies JC, Cunningham S, Harris WT, et al. Safety, pharmacokinetics, and pharmacodynamics of ivacaftor in patients aged 2-5 years with cystic fibrosis and a CFTR gating mutation (KIWI): an open-label, single-arm study. Lancet Respir Med. 2016;4:107-15.

26. Rowe SM, Daines C, Ringshausen FC, et al. Tezacaftor-ivacaftor in residual-function heterozygotes with cystic fibrosis. $N$ Eng/ J Med. 2017;377:2024-35.

27. Bessonova L, Volkova N, Higgins $M$, et al. Data from the US and UK cystic fibrosis registries support disease modification by CFTR modulation with ivacaftor. Thorax. 2018;73:731-40.

28. Flume PA, Liou TG, Borowitz DS, et al. Ivacaftor in subjects with cystic fibrosis who are homozygous for the F508del-CFTR mutation. Chest. 2012:142:718-24.

29. Dalemans W, Barbry P, Champigny G, et al. Altered chloride ion channel kinetics associated with the delta F508 cystic fibrosis mutation. Nature. 1991;354:526-8.

30. Bobadilla JL, Macek M Jr, Fine JP, Farrell PM. Cystic fibrosis: a worldwide analysis of CFTR mutations--correlation with incidence data and application to screening. Human mutation. 2002; 19:575-606.

31. Vertex Pharmaceuticals Inc. ORKAMBI (lumacaftor/ivacaftor) [package insert]. Boston, MA. 2015.

32. Van Goor F, Hadida S, Grootenhuis PD, et al. Correction of the F508del-CFTR protein processing defect in vitro by the investigational drug VX-809. Proc Natl Acad Sci USA. 2011;108:18843-8

33. Loo TW, Bartlett MC, Clarke DM. Corrector VX-809 stabilizes the first transmembrane domain of CFTR. Biochem Pharmacol. 2013;86:612-9

34. Clancy JP, Rowe SM, Accurso FJ, et al. Results of a phase lla study of VX-809, an investigational CFTR corrector compound, in subjects with cystic fibrosis homozygous for the F508del-CFTR mutation. Thorax. 2012:67:12-8.

35. Boyle MP, Bell SC, Konstan MW, et al. A CFTR corrector (lumacaftor) and a CFTR potentiator (ivacaftor) for treatmen of patients with cystic fibrosis who have a phe508del CFTR mutation: a phase 2 randomised controlled trial. Lancet Respir Med. 2014;2:527-38.

36. Wainwright CE, Elborn JS, Ramsey BW, et al. Lumacaftor-ivacaftor in patients with cystic fibrosis homozygous for Phe508del CFTR. N Eng/ J Med. 2015;373:220-31.

37. Elborn JS, Ramsey BW, Boyle MP, et al. Efficacy and safety of lumacaftor/ivacaftor combination therapy in patients with cystic fibrosis homozygous for Phe508del CFTR by pulmonary function subgroup: a pooled analysis. Lancet Respir Med. 2016;4:617-26

38. Konstan MW, McKone EF, Moss RB, et al. Assessment of safety and efficacy of long-term treatment with combination lumacaftor and ivacaftor therapy in patients with cystic fibrosis homozygous for the F508del-CFTR mutation (PROGRESS): a phase 3, extension study. Lancet Respir Med. 2017;5:107-18.

39. Rowe SM, McColley SA, Rietschel E, et al. Lumacaftor/ivacaftor treatment of patients with cystic fibrosis heterozygous for F508del-CFTR. Ann Am Thorac Soc. 2017;14:213-9.

40. Vertex Pharmaceuticals Inc. SYMDEKO (tezacaftor/ivacaftor) [package insert]. Boston, MA. 2018.
41. Donaldson SH, Pilewski JM, Griese M, et al. Tezacaftor/ivacaftor in subjects with cystic fibrosis and F508del/F508del-CFTR or F508del/G551D-CFTR. Am J Respir Crit Care Med. 2018;197: $214-24$

42. Taylor-Cousar JL, Munck A, McKone EF, et al. Tezacaftor-ivacaftor in patients with cystic fibrosis homozygous for Phe508del. N Eng J Med. 2017;377:2013-23.

43. Taylor-Cousar JL, Mall MA, Ramsey BW, et al. Clinical development of triple-combination CFTR modulators for cystic fibrosis patients with one or two F508del alleles. ERJ Open Res. 2019;5. pii: 00082-2019.

44. Grootenhuis P, Van Goor F, Hadida S, et al. Discovery and biologica profile of next-generation CFTR correctors (abstract). Pediatr Pulmonol. 2016:51:S263.

45. Davies JC, Colombo C, Tullis E, et al. Preliminary safety and efficacy of triple combination CFTR modulator regimens in cystic fibrosis. Journal of Cystic Fibrosis. 2018;17S3:S3.

46. Keating D, Marigowda G, Burr L, et al. VX-445-tezacaftor-ivacaftor in patients with cystic fibrosis and one or two Phe508del alleles. N Engl J Med. 2018;379:1612-20.

47. Davies JC, Moskowitz SM, Brown C, et al. VX-659-tezacaftorivacaftor in patients with cystic fibrosis and one or two Phe508del alleles. N Eng/ J Med. 2018:379:1599-611.

48. Vertex Pharmaceuticals Inc. Two phase 3 studies of the triple combination of VX-659 tezacaftor and ivacaftor met primary endpoint of improvement in lung function (ppFEV1) in people with cystic fibrosis, 2018. Available at: https://investors.vrtx.com/ news-releases/news-release-details/two-phase-3-studies-triplecombination-vx-659-tezacaftor-and (accessed January 23, 2020).

49. Vertex Pharmaceuticals Inc. Two phase 3 studies of the triple combination of $\mathrm{VX}-445$ tezacaftor and ivacaftor met primary endpoint of improvement in lung function (ppFEV1) in people with cystic fibrosis. 2019. Available at: https://investors.vrtx.com news-releases/news-release-details/correcting-and-replacingtwo-phase-3-studies-triple-combination (accessed January 23, 2020)

50. Middleton PG, Mall MA, Drevinek P, et al. Elexacaftor-tezacaftorivacaftor for cystic fibrosis with a single Phe508del allele. N Eng/ J Med. 2019;381:1809-19.

51. Heijerman HGM, MCKone EF, Downey DG, et al. Efficacy and safety of the elexacaftor plus tezacaftor plus ivacaftor combination regimen in people with cystic fibrosis homozygous for the F508del mutation: a double-blind, randomised, phase 3 tria Lancet. 2019;394:1940-8.

52. Vertex Pharmaceuticals Inc. TRIKAFTA (elexacaftor, tezacaftor and ivacaftor tablets; ivacaftor tablets) [package insert]. Boston, MA. 2019.

53. Vertex Pharmaceuticals Inc, FDA accepts new drug application for VX-445 (elexacaftor), tezacaftor and ivacaftor combination treatment, 2019. Available at: https://investors.vrtx.com/newsreleases/news-release-details/fda-accepts-new-drug-applicationvx-445-elexacaftor-tezacaftor (accessed January 23, 2020)

54. Vertex Pharmaceuticals Inc, FDA approves TRIKAFTA (elexacaftor/ tezacaftor/ivacaftor and ivacaftor) to treat the underlying cause of cystic fibrosis in people ages 12 and older who have at least one F508del mutation, 2019 . Available at: https://investors vrtx. $\mathrm{com} /$ news-releases/news-release-details/adding-multimedia-fdaapproves-trikafta (accessed January 23, 2020).

55. Andersen DH. Cystic fibrosis of the pancreas and its relation to celiac disease: a clinical and pathologic study. Am J Dis Child. 1938;56:344-99.

56. Schrijver I, Pique L, Graham S, et al. The spectrum of CFTR variants in nonwhite cystic fibrosis patients: implications for molecular diagnostic testing. J Mol Diagn. 2016;18:39-50. 